BRAZZILIAN JOURNAL

OF MEDICAL AND BIOLOGICAL RESFARCH

www.bjournal.com.br
ISSN 0100-879X

Volume 44 (11) 1070-1193 November 2011

BIOMEDICAL SCIENCES

AND

CLINICAL INVESTIGATION

Braz J Med Biol Res, November 2011, Volume 44(11) 1112-1117

doi: 10.1590/S0100-879X2011007500120

Polymorphism of the aryl-hydrocarbon receptor gene in intron 10 of human cancers

M. Rocas, E. Jakubauskiene and A. Kanopka

The Brazilian Journal of Medical and Biological Research is partially financed by
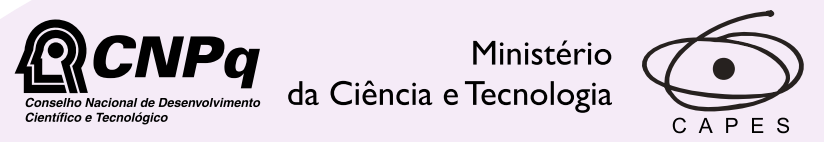

Ministério da Educação
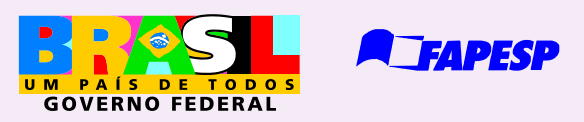

Institutional Sponsors
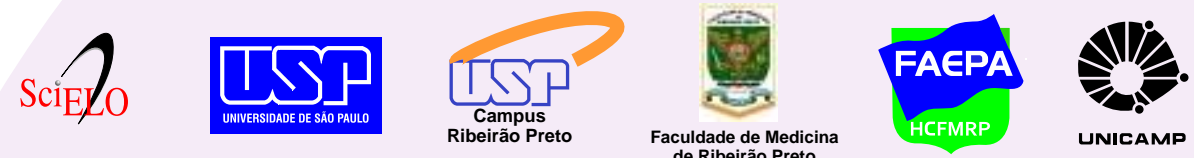

UNICAMP
Ф SHIMADZU

Explore High - Performance MS Orbitrap Technology In Proteomics \& Metabolomics

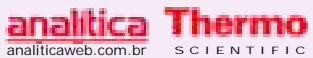




\title{
Polymorphism of the aryl-hydrocarbon receptor gene in intron 10 of human cancers
}

\author{
M. Rocas, E. Jakubauskiene and A. Kanopka \\ Department of Immunology and Cell Biology, Institute of Biotechnology, \\ Vilnius University, Vilnius, Lithuania
}

\begin{abstract}
Polychlorinated dibenzo-p-dioxins (PCDDs) and related halogenated aromatic hydrocarbons (e.g., PCDFs), often called "dioxins", are ubiquitously present environmental contaminants. Some of them, notably 2,3,7,8-tetrachlorodibenzo- $p$-dioxin (TCDD), are among the most toxic synthetic compounds known. The biological effects of dioxins are mediated via the aryl hydrocarbon receptor (AhR). Mutations in the AhR transactivation domain are linked to sensitivity to the acute lethality of TCDD. We present here a study of AhR gene polymorphism in normal and cancer human tissues affecting pre-mRNA splicing in the AhR gene-coding transactivation domain region (exon 10, intron 10, exon 11 region), previously shown to be associated with AhR dysfunction. We tested 126 pairs of normal and cancer tissue samples from liver, lung, stomach, kidney, mucous, breast, and pancreas of 49 males and 77 females (45-70 years of age). We used in vitro splicing assay, RT-PCR and sequencing methods. Our results showed that in an in vitro system it is possible to reconstitute cellular pre-mRNA splicing events. Tested cancer tissues did not contain mutations in the AhR transactivation domain region when the DNA sequences were compared with those from normal tissues. There were also no differences in AhR mRNA splice variants between normal and malignant breast tissues and no polymorphisms in the studied regions or cDNA.
\end{abstract}

Key words: Ah receptor; Polymorphism; Splicing; Cancer; Dioxins

\section{Introduction}

One of the characteristics of eukaryotic genes that distinguish them from their prokaryotic counterparts is the production of large pre-mRNA that contains intervening non-coding sequences (introns). Intervening sequences in eukaryotic genes are removed from nuclear pre-mRNA in a well-defined pathway termed splicing. Pre-mRNA splicing proceeds in the nucleus and occurs co-transcriptionally. The coding sequences (exons) are joined to generate the mature mRNA that is exported to the cytoplasm and translated into protein. The efficiency of pre-mRNA splicing is critically dependent upon the accuracy of cleavage and rejoining. The vast majority of human genes contain introns, and most pre-mRNAs undergo splicing. Alterations in pre-mRNA splicing cause changes in protein function, cellular sensitivity to some agents and, in some cases, cell death (1-3). It is not surprising that disruption of normal splicing patterns can cause or modify human disease $(1,4)$. Mutations within splice sites represent about $15 \%$ of known point mutations causing human genetic diseases (5). For example, mutations disrupting the normal PKHD1 gene splicing cause autosomal recessive polycystic disease (6). For many genes in mammals, changes in alternative splicing patterns are associated with tumor development and metastasis $(2,5)$. For instance, it was recently shown that switching between splice isoforms of CD44 is associated with epithelial-to-mesenchymal transition and progression of breast cancer (7). Also a splice-variant of osteopontin, osteopontin-c, is specifically expressed in ovarian cancer cells and is involved in the progression of ovarian cancer (8). Therefore, alternatively spliced transcripts may be extremely useful as markers of certain types of cancer, since difference in usage of alternatively spliced transcript variants between normal and tumor tissue might be more pronounced than alterations in the general levels of gene expression (2).

Polychlorinated dibenzo-p-dioxins (PCDDs) and polychlorinated dibenzofuranes (PCDFs) are ubiquitously present environmental contaminants with well-established potent toxicity. In the cell, dioxins induce transcription of a battery of target genes encoding xenobiotic metabolizing

Correspondence: A. Kanopka, Department of Immunology and Cell Biology, Institute of Biotechnology, Vilnius University, Graiciuno 8, 02241 Vilnius, Lithuania. Fax: +370-5-260-2116. E-mail: kanopka@ibt.It

Received March 8, 2011. Accepted September 5, 2011. Available online September 16, 2011. Published November 14, 2011. 
enzymes, such as the glutathione S-transferase Ya subunit, and transcriptionally regulate the expression of growth modulatory genes for interleukin-1 $\beta$ and plasminogen activator inhibitor-2 (9).

In animals, the toxic effects of dioxins lead to thymic wasting and immune suppression, causing epithelial disorders and tumor promotion (10). In humans, a wide variety of health effects and cancer development $(11,12)$ have been linked to exposure to dioxins.

The biological effects of dioxins are mediated by a ligand-dependent aryl hydrocarbon (or dioxin) receptor (AhR) belonging to the basic helix-loop-helix (bHLH) - PAS [Per, aryl hydrocarbon receptor nuclear translocator (ARNT), Sim] family of transcription factors. Upon ligand binding, series of events lead to the translocation of AhR into the nucleus, where it dimerizes with another bHLH/PAS protein, the ARNT (13). The AhR-ARNT-dioxin complex then binds to DNA at one or several specific sites designated as dioxin response elements acting as transcriptional enhancers and induces transcription of CYP1A1 and probably other genes $(9,14)$.

The AhR plays a critical role in response to xenobiotic compounds. In laboratory animals, genetic variants in AhR mRNA lead to substantial differences in sensitivity to the biochemical and toxic effects of dioxins. A striking difference in susceptibility to acute lethality between two rat strains, Long-Evans (L-E) and Han/Wistar (H/W), has been demonstrated. Differences between L-E rats and H/W rats in sensitivity to 2,3,7,8-tetrachlorodibenzo-p-dioxin (TCDD) are correlated with differential AhR mRNA processing at the exon 10 region due to an invariant GT nucleotide at the beginning of intron 10 (15). In humans, a wide variety of health effects have been linked to exposure to dioxins, including mood alterations, reduced cognitive performance, diabetes, dental defects, endometriosis, decreased testosterone, and cancer (16-18).

In the present study, we have determined if some human cancers have mutations in the AhR gene, which might affect its pre-mRNA processing in the region that is implicated in transactivation of AhR-target genes. We also determined whether the mRNAsplicing pattern of AhR mRNA is affected in breast tumor tissue.

\section{Material and Methods}

\section{Material}

All salts, glycerol, polyvinyl alcohol, phenol, agarose, and ATP were purchased from Roth (Germany), and phosphocreatine, polyvinyl alcohol, PMSF, acrylamide, and bisacrylamide were from Sigma (Sigma-Aldrich Chemie $\mathrm{GmbH}$, Germany). All restriction endonucleases, DNA ligase, proteinase K, T7 RNA polymerase, Taq DNA polymerase, and pBluescript II KS(+) plasmid DNA were from Fermentas (Lithuania), TRIzol reagent was from Invitrogen (USA), RT-PCR kit was from ABgene (UK), and [ $\left.{ }^{32} \mathrm{P}\right]-C T P$ was from Hartman Analytic (Germany). All DNA primers were purchased from Metabion (Germany), and ethanol was from Vilniaus degtine (Lithuania).

\section{Escherichia coli strains}

The strains used were E. coli DH5 $\alpha\left[\mathrm{F}^{-},(\varphi 80\right.$ dlacZ $\triangle \mathrm{M} 15)$, recA1, endA1, gyrA96, thi-1, hsdR17( $\left.r_{k}^{-} m_{k}{ }^{+}\right)$, supE44, relA1, deoR, $\Delta$ (lacZYA-argF)U169] (Fermentas).

\section{Cloning}

DNA constructs were assembled from the AhR gene spanning exon 10-intron 10 and intron 10-exon 11 from L-E (wild type) and H/W (mutant) Rattus norvegicus rat strains (NCBI Accession Nos. AF082124, AF082125, and AF082126) by PCR using appropriate primer pairs: P1-P2 - constructs 5' fragment [P1: 5'-d(AAGGAGAGCTCCATG GTCAGTCCTCAGGCG)-3'; P2: 5'-d(TTCCTAAGCTTCTG CCTAAAAGGATTCTGAC)-3'] and P3-P4 - constructs 3' fragment [P3: 5'-d(AAGGAAAGCTTGATATCGAATTCCTG CAGCC)-3'; P4:5'-d(TTCCTCTCGAGTCTGAAGGTGGCC AATGC)-3']. PCR fragments [wild-type (AhR-10 w.t. $)$ and mutant (AhR-10 mut.)] through Sacl/HindIII and HindlII/Xhol restriction endonuclease sites, respectively, were cloned into pBluescript II KS (+) plasmid DNA.

\section{T7 transcription and pre-mRNA splicing in vitro}

Labeled pre-mRNAs from the plasmids were generated in an in vitro transcription reaction using T7 RNA polymerase and [ $\left.{ }^{32} \mathrm{P}\right]-\mathrm{CTP}$ and the transcription reaction products were purified (19). Nuclear extracts from HeLa cells and in vitro splicing reactions were performed as described previously $(19,20)$. Splicing reactions $(25 \mu \mathrm{L})$ contained: $\approx 10 \mathrm{fmol}$ in vitro transcribed, capped and [ $\left.{ }^{32} \mathrm{P}\right]$-labeled pre-mRNA, $20 \%$ nuclear extract, $2.6 \%(\mathrm{w} / \mathrm{v})$ polyvinyl alcohol, $2.8 \mathrm{mM}$ $\mathrm{MgCl}_{2}$, 2 mM ATP, $20 \mathrm{mM}$ phosphocreatine, and buffer D (20\%, w/v) glycerol, 20 mM HEPES, pH 7.9, 100 mM KCl, 0.2 mM EDTA, 0.5 mM DTT, 0.3 mM PMSF). Reactions were incubated for 0.5 to $2 \mathrm{~h}$ at $30^{\circ} \mathrm{C}$ followed by proteinase $\mathrm{K}$ digestion $(40 \mu \mathrm{g})$ for $30 \mathrm{~min}$ at $37^{\circ} \mathrm{C}$, extracted with phenolchloroform and ethanol precipitated. Pre-mRNA splicing reaction products were separated on $8 \%$ denaturating polyacrylamide gels.

\section{Preparation of DNA from mammalian tissues}

DNA from human normal and cancer tissues was prepared according to a published protocol (21). PCR was performed using primers P5 (5'-d(CACGTGGGTCAGAT GCAGTACAATCC)-3') and P6 (5'-d(GGTTTCAGAGGC ATTGTAAGAAAGCAC)-3') or P7 (5'-d(CAAGACAGAGC ATTTTCTTTTGGTAACTTG)-3') and P8 (5'-d(GATGATG AAGTGGCTGAAGATGTGTGG)-3'). Reaction products were sequenced by di-deoxy sequencing methods using a manual cycle reader DNA sequencing kit (Fermentas, Lithuania) or at an institutional sequencing center. The 
sequences were compared using the web-based Basic Local Alignment Search Tool (BLAST) software bl2seq (http://blast.ncbi.nlm.nih.gov/Blast.cgi), and any type of polymorphism was screened.

\section{Isolation of total RNA from tissues}

Total RNA from human normal and cancer tissues was isolated using TRIzol reagent. RT-PCR assays contained $1 \mu \mathrm{g}$ RNA and $10 \mathrm{pmol}$ each of primers: P9 (5'-d(CAGCGCCAACATCACCTAC)-3') and P10 (5'-d(GA AGCACCTCTCCATTAAAGG)-3') or P11 (5'-d(CCTTTAAT GGAGAGGTGCTTC)-3') and P12 (5'-d(CGTAAATGCTC TGTTCCTTCC)-3'). RT-PCR was performed according to the ABgene protocol. Products were sequenced.

\section{Results}

Due to mutations in primary gene sequence, pre-mRNA splicing patterns can serve as markers of the altered cellular state associated with disease even when they are not in the primary pathway of the disease mechanism and have the potential to provide diagnostic and prognostic information.

Before screening for mutations in the AhR gene transactivation domain (intron 10 region) or AhR mRNA in human cancers, we examined whether it is possible, using an in vitro splicing system, to reproduce the splicing shift in the AhR intron 10 region, which occurs in rat cells. As the model pre-mRNAs in splicing assays, we used rat AhR constructs from the L-E rat strain, which contains the wild-type $A h R$ receptor gene, or the mutant $\mathrm{H} / \mathrm{W}$ rat strain, containing mutation in the AhR gene (Figure 1). AhR-10 w.t. and AhR-10 $10_{\text {mut. }}$ pre-mRNAs were generated in an in vitro transcription reactions and used in an in vitro splicing reaction in HeLa nuclear extracts. The time course of the splicing reactions showed that the AhR- 10 w.t. construct, as expected, yielded a product of 205 nucleotides (nt; Figure 2 , lanes 6-10). The AhR-10 mut. construct, where the G nucleotide at the 5 ' splice site is mutated to A, yielded a 234-nt product (Figure 2, lanes 1-5). The 234-nt band was cut out from the gel and eluted and subjected to reverse transcription combined with PCR (RT-PCR). The primary DNA structure of the fragment obtained was determined by sequencing. The sequencing results revealed that in pre-
mRNA, containing the mutated 5' splice site, the aberrant splice site located 29 nt upstream of a normal splice site was utilized. The result obtained demonstrates that cellular events can be reconstituted in this in vitro system.

The human AhR gene encompasses 47,146 nt and is located on chromosome 7. There are 12 exons, which encode an AhR mRNA (5483 nt). At present, most studies on AhR gene polymorphism are conducted on exonic sequence polymorphism (22). The exon 10 region of the Ahr gene encodes one of the major protein regions that is implicated in the transactivation of target genes $(11,22)$. As the mutations in the AhR transactivation domain (NCBI accession No. NM_001621) determine a clear difference in susceptibility to the acute lethality of TCDD (15), it is possible to expect that aberrant processing of AhR mRNA in intron 10 regions might lead to changes in the efficiency of removal of non-coding sequences, to abnormal AhR protein function and to cancer development in humans.

In the next step of our study, we searched for any possible mutations that might influence AhR pre-mRNA processing in the intron 10 regions, or intron removal efficiency by comparing sequences of DNA isolated from normal and cancer human tissues provided by the Lithuanian Cancer Center. The normal and cancer tissues tested were (126 sample pairs): liver $=15$, lung $=26$, stomach $=15$, kidney $=15$, mucous $=15$, breast $=25$, and pancreas $=15$. After isolation of DNA from these tissues, PCR was performed using specifically designed primers (see Figure 3), and the PCR products were sequenced. Sequence analysis of the PCR product fragments obtained from DNA isolated from normal and cancer tissues of different individuals revealed no differences in primary structure of the AhR gene transactivation domain (intron 10) region. These data indicate that there were no changes in primary DNA sequence that might affect AhR pre-mRNA processing in the cancer types studied.

Pre-mRNA splicing variants have been implicated in the progression and spreading of several human tumors (23). Altered splicing patterns can also serve as markers of the altered cellular state associated with disease or, e.g., a toxic environment. Therefore, detection of altered splicing patterns has the potential to provide diagnostic and prognostic information.

Since dioxins are lipid-soluble compounds, they might

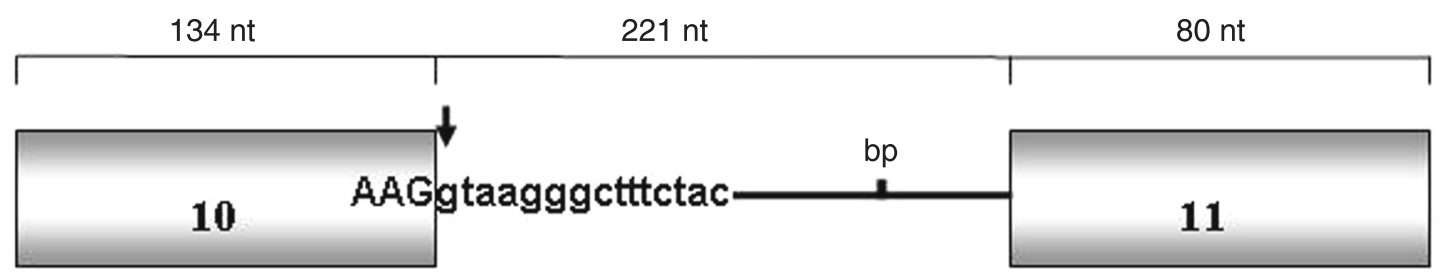

Figure 1. Rat AhR (intron 10 region) pre-mRNA constructs constructed for pre-mRNA splicing reactions in vitro. The arrow indicates the location of the point mutation detected in the $\mathrm{H} / \mathrm{W}$ rat strain. AhR = aryl hydrocarbon receptor; bp $=$ branch point nucleotide. Intron and exon sizes in nucleotides (nt) of constructs are indicated. 


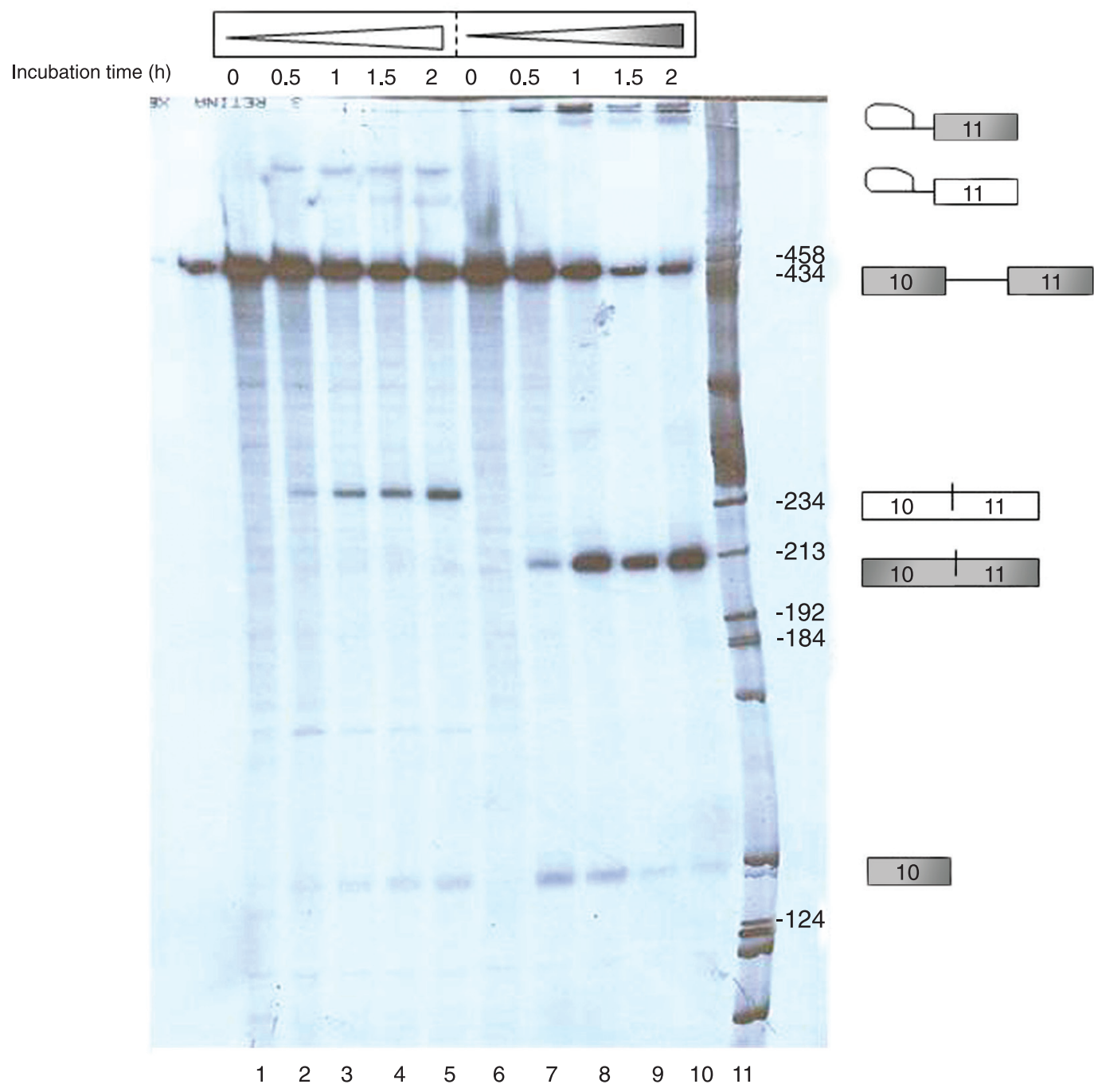

Figure 2. Time course of the in vitro splicing reaction of the wild-type AhR-10 (lanes 6-10) and the mutant AhR-10 (lanes 1-5) constructs in HeLa cell nuclear extracts. Lanes 1 and $6=$ incubation time in HeLa cell nuclear extract $0 \mathrm{~h}$; lanes 2 and $7=0.5 \mathrm{~h}$; lanes 3 and $8=1 \mathrm{~h}$; lanes 4 and $9=1.5 \mathrm{~h}$; lanes 5 and $10=2 \mathrm{~h}$. Lane $11=\mathrm{RNA}$ size marker. Ahr $=$ aryl hydrocarbon receptor.

accumulate and be most toxic in tissues that are rich in fat. To test the possibility that dioxins might alter the splicing pattern, we chose to analyze polymorphism of AhR mRNA in the breast tissues, which are rich in fat. To this end, total RNA from normal breast tissue and breast cancer tissue was isolated using primers specific for AhR mRNA (Figure 4), converted to cDNA by RT-PCR and sequenced. Comparison of sequences revealed no changes in nucleotide sequence of AhR cDNA from normal breast tissue and breast cancer tissue. These results suggest that the primary structure of the human AhR protein is highly conserved.

\section{Discussion}

In addition to its roles in sensing toxicity and as a mediator of the induction of drug-metabolizing enzymes, AhR is also involved in carcinogenesis. Shimizu et al. (12) have demonstrated thatAhR-deficient mice treated with benzo[a] pyrene, a widely distributed environmental carcinogen, do not develop subcutaneous tumors at the site of injection, while all AhR-positive mice bearing +/+ and +/- genotypes do. Their finding indicates that AhR plays an important role in cancer development in animal models. Recently, it has been shown that polymorphisms in exonic sequence of the 
A

\section{Primer 5}

EXON 10 - CACGTGGGTCAGATGCAGTACAATCCAGACTGCCAGGCCAACAGGCATTTTTAAA CAAGgtaagggtgttatcaaactgaattaaatctttcagtgattcttttaccttatagacatgttacacatttttatgtcagctgattttaatcggttatctacagcattcatgg agacagcattttttattatatctgtgactaccttttttttttt acaagcctgtacttttccagtatgttactaat atatcattcacctgattcatgagggagaaagatatcaaggaat gaagcaacatgaat gtgtccttaatataagatgacctaaattaccaggtgaaattagtttttagaagtattttgtttaaaat agataatttggtttattctttctggattctttaaaaat tatatggctatttaaaattcaagctttaaaaaacgtattgcaatttttaagaataatttcttcaaagctctattttttatatatgtttgaaattgagttaaactcaaatttaagtgtgct ttcttacaatgcetctgaaacc - intron 10

Primer 6

B

Primer 7

intron 10 - caagacagagcatttctttggtaacttgattctgtaattagaactatgcttcagtagtattcattaattaaagtatattatgaataagaaatcggttaaga aaatcatacctgtggtgtaatattttaggaatatttagaaacatttagaaaatcagaagctgcctttttggaaagttttgcattccagtgtaagattcctgaatataatcaattcatt gatacctataacttcaaacctggtcaaagcaaacctacttatgatagagaatatttaactgtaatttgtgacttgtgctgtttgacccatcaattcctacctgacccaaaggcaatct gaaatgagacagctgcatcactcatctgtaccatgttcaaaatttcctgtcaaattaatggaaaacaggttataaatgcaactaatacataatatgtcctcagtatgtcttcactgt gtgaaagatttaaaatttagcaacagtaaaggaacttgaagtttacaactctcaggggtaagattttaaaaatacatgttaat gttatttactggcttaagatacttggaagatctatt c caataagttgcatcaccatttttgttttcagTTTCAGAATGGAGTTTTAAATGAAACATATCCAGCTGAATTAAATAACATA AATAACACTCAGACTACCACACATCTTCAGCCACTTCATCATC - EXON 11 Primer 8

Figure 3. AhR gene intron 10 region sequences spanned by designed primer pairs P5-P6 and P7-P8. A, Primer pair P5-P6 spanning a 494-bp fragment (3' end of exon 10-5' end of intron 10). B, Primer pair P7-P8 spanning a 644-bp fragment ( 3 ' end of intron 10-5' end of exon 11). Exonic sequences are indicated in upper case and intronic sequences in lower case letters. Primer sequences are shown in bold. Ahr = aryl hydrocarbon receptor.

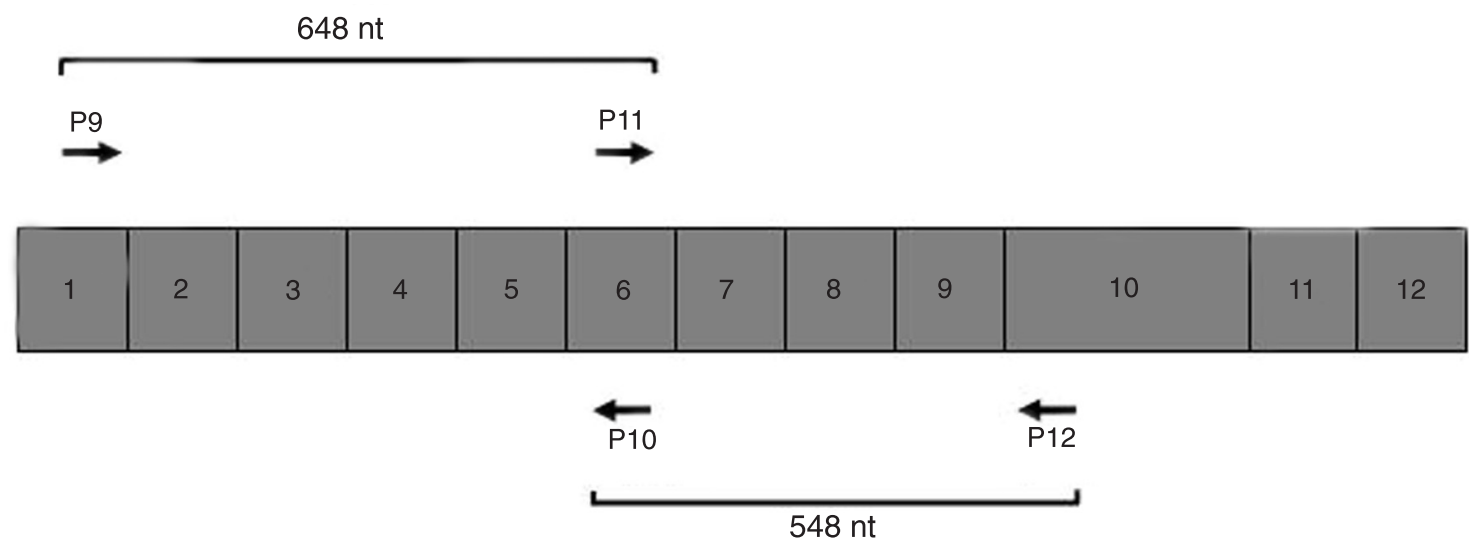

Figure 4. Primer pairs (P9-P10 and P11-P12) designed for AhR cDNA polymorphism studies. The spanned fragment size (nucleotides, nt) is indicated. Ahr $=$ aryl hydrocarbon receptor.

AhR receptor are associated significantly with risk of nonHodgkin lymphoma and that these polymorphisms significantly increase the risk of lung cancer in humans $(24,25)$.

In the present study, we have searched for polymorphisms in the sequence of the processing region of the AhR gene (exon 10-intron 10-exon 11) in various types of human cancers. Our sequence analyses did not reveal differences in this region, including the sequence of intron
10, which might affect intron 10 region splicing or the efficiency in intron removal in cancers. Our results indicate that the primary structure of the human AhR protein is highly conserved in the cancer types studied. The high degree of conservation in the primary structure of the AhR in humans suggests that this protein has critical life functions (15). Many of the genes affected by exposure to AhR ligands in animal models or in cell culture are involved in critical events 
such as cell proliferation, cell signaling and apoptosis. The list of genes whose expression is altered by AhR ligands is continually expanding (22).

As we examined only a limited set of different cancer types (phenotypes), we cannot completely exclude the possibility that, at the genomic level, there might be changes in the AhR gene intron 10 region of other cancer types. Therefore, it seems that the appearance of the studied cancers was not due to the genetic alterations within the intron 10 region. It is likely that the role of the dioxin receptor in cancer formation in humans is indirect, and therefore it remains to be determined which cellular pathways are affected by exposure to dioxins.

\section{References}

1. Cooper TA, Wan L, Dreyfuss G. RNA and disease. Cell 2009; 136: $777-793$.

2. David CJ, Manley JL. Alternative pre-mRNA splicing regulation in cancer: pathways and programs unhinged. Genes Dev 2010; 24: 2343-2364.

3. Jiang $\mathrm{ZH}, \mathrm{Wu} \mathrm{JY}$. Alternative splicing and programmed cell death. Proc Soc Exp Biol Med 1999; 220: 64-72.

4. Yu J, Nagarajan S, Ueda E, Knez JJ, Petersen RB, Medof ME. Characterization of alternatively spliced PIG-A transcripts in normal and paroxysmal nocturnal hemoglobinuria cells. Braz J Med Biol Res 1994; 27: 195-201.

5. Faustino NA, Cooper TA. Pre-mRNA splicing and human disease. Genes Dev 2003; 17: 419-437.

6. Menezes LF, Onuchic LF. Molecular and cellular pathogenesis of autosomal recessive polycystic kidney disease. Braz J Med Biol Res 2006; 39: 1537-1548.

7. Brown RL, Reinke LM, Damerow MS, Perez D, Chodosh LA, Yang $\mathrm{J}$, et al. CD44 splice isoform switching in human and mouse epithelium is essential for epithelial-mesenchymal transition and breast cancer progression. J Clin Invest 2011; 121: 1064-1074.

8. Tilli TM, Franco VF, Robbs BK, Wanderley JL, da Silva FR, de Mello KD, et al. Osteopontin-c splicing isoform contributes to ovarian cancer progression. Mol Cancer Res 2011; 9: 280293

9. Marlowe JL, Puga A. Aryl hydrocarbon receptor, cell cycle regulation, toxicity, and tumorigenesis. J Cell Biochem 2005; 96: 1174-1184.

10. Pohjanvirta R, Tuomisto J. Short-term toxicity of 2,3,7,8-tetrachlorodibenzo-p-dioxin in laboratory animals: effects, mechanisms, and animal models. Pharmacol Rev 1994; 46: 483-549.

11. Cauchi S, Stucker I, Solas C, Laurent-Puig P, Cenee S, Hemon $D$, et al. Polymorphisms of human aryl hydrocarbon receptor $(\mathrm{AhR})$ gene in a French population: relationship with CYP1A1 inducibility and lung cancer. Carcinogenesis 2001; 22: 18191824.

12. Shimizu $Y$, Nakatsuru $Y$, Ichinose $M$, Takahashi $Y$, Kume $H$, Mimura J, et al. Benzo[a]pyrene carcinogenicity is lost in mice lacking the aryl hydrocarbon receptor. Proc Natl Acad Sci U S A 2000; 97: 779-782.

13. Gradin K, McGuire J, Wenger RH, Kvietikova I, fhitelaw ML, Toftgard R, et al. Functional interference between hypoxia and dioxin signal transduction pathways: competition for recruitment

\section{Acknowledgments}

Research supported by the Lithuanian State Science and Studies Foundation (grant \#T-44/04). We thank Dr. Raimo Pohjanvirta (Department of Food and Environmental Hygiene, Faculty of Veterinary Medicine, University of Helsinki, Helsinki, Finland) for providing Long-Evans (L-E) and Han/Wistar $(\mathrm{H} / \mathrm{W})$ rat strain AhR clones, and Dr. A. Meskauskas for providing normal and malignant human tissues. We also thank Dr. Simonas Laurinavicius for valuable discussion of this manuscript.

of the Arnt transcription factor. Mol Cell Biol 1996; 16: 5221 5231.

14. Rowlands JC, McEwan IJ, Gustafsson JA. Trans-activation by the human aryl hydrocarbon receptor and aryl hydrocarbon receptor nuclear translocator proteins: direct interactions with basal transcription factors. Mol Pharmacol 1996; 50: 538-548.

15. Pohjanvirta R, Wong JM, Li W, Harper PA, Tuomisto J, Okey AB. Point mutation in intron sequence causes altered carboxylterminal structure in the aryl hydrocarbon receptor of the most 2,3,7,8-tetrachlorodibenzo-p-dioxin-resistant rat strain. Mol Pharmacol 1998; 54: 86-93.

16. Alaluusua S, Lukinmaa PL, Torppa J, Tuomisto J, Vartiainen T. Developing teeth as biomarker of dioxin exposure. Lancet 1999; 353: 206.

17. Mayani A, Barel S, Soback S, Almagor M. Dioxin concentrations in women with endometriosis. Hum Reprod 1997; 12: 373-375.

18. Steenland K, Bertazzi P, Baccarelli A, Kogevinas M. Dioxin revisited: developments since the 1997 IARC classification of dioxin as a human carcinogen. Environ Health Perspect 2004; 112: 1265-1268.

19. Kreivi JP, Zerivitz K, Akusjarvi G. Sequences involved in the control of adenovirus L1 alternative RNA splicing. Nucleic Acids Res 1991; 19: 2379-2386.

20. Dignam JD, Lebovitz RM, Roeder RG. Accurate transcription initiation by RNA polymerase II in a soluble extract from isolated mammalian nuclei. Nucleic Acids Res 1983; 11: 1475-1489.

21. Strauss WM. Preparation of genomic DNA from mammalian tissue. In: Ausubel FM, Brent R, Kingston RE, Moore DD, Seidman JG, Smith JA, et al. (Editors), Current protocols in molecular biology. New York: John Wiley \& Sons, Inc.; 1998.

22. Harper PA, Wong JY, Lam MS, Okey AB. Polymorphisms in the human AH receptor. Chem Biol Interact 2002; 141: 161-187.

23. Caballero OL, de Souza SJ, Brentani RR, Simpson AJ. Alternative spliced transcripts as cancer markers. Dis Markers 2001; 17: $67-75$.

24. Chen D, Tian T, Wang H, Liu H, Hu Z, Wang Y, et al. Association of human aryl hydrocarbon receptor gene polymorphisms with risk of lung cancer among cigarette smokers in a Chinese population. Pharmacogenet Genomics 2009; 19: 25-34.

25. $\mathrm{Ng} \mathrm{CH}$, Janoo-Gilani R, Sipahimalani P, Gallagher RP, Gascoyne RD, Connors JM, et al. Interaction between organochlorines and the AHR gene, and risk of non-Hodgkin lymphoma. Cancer Causes Control 2010; 21: 11-22. 